\title{
Author Correction: High-performance, polymer-based direct cellular interfaces for electrical stimulation and recording
}

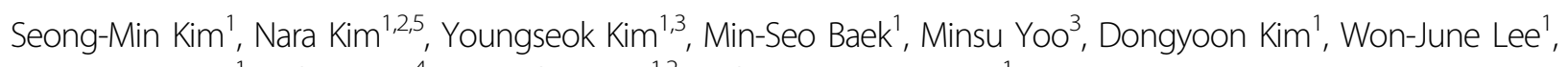
Dong-Hee Kang ${ }^{1}$, Sohee Kim ${ }^{4}$, Kwanghee Lee ${ }^{1,2}$ and Myung-Han Yoon ${ }^{1}$

Correction to: NPG Asia Materials (2018) https:/doi.org/ 10.1038/s41427-018-0014-9 published online on 16 April 2018

The original version of this article unfortunately contained an error in the author name. The spelling of the Min-Seo Baek's name was incorrect.

Additionally, the Author Contributions section in this Article was incomplete.

"S.-M.K., N.K., Y.K., K. L. and M.-H.Y. designed all the experiments. S.-M.K., N.K. and Y.K. prepared the PEDOT:PSS films. S.-M.K., N.K., M.-S.B. and W.-J.L. performed the film characterizations. S.-M.K., Y.K., M.-S.B., D.H.K., M.Y. and D.K. conducted biological experiments. Y.K. fabricated and characterized the multi-electrode arrays (MEAs) and measured the cell potentials using the MEAs. S.-M.K, N.K, Y.K, K. L. and M.-H.Y wrote the manuscript. All authors commented on the manuscript." now reads:

"S.-M.K., N.K., Y.K., K. L. and M.-H.Y. designed all the experiments. S.-M.K., N.K. and Y.K. prepared the PEDOT:PSS films. S.-M.K., N.K., M.-S.B. and W.-J.L. performed the film characterizations. S.-M.K., Y.K., M.-S. B., D.-H.K., M.Y. and D.K. conducted biological experiments. Y.K. fabricated and characterized the multi-electrode arrays (MEAs) and measured the cell potentials using the MEAs. S.-M.K, N.K, Y.K, K. L. and M.-H.Y wrote the manuscript. S.K. contributed to discussions throughout the project. All authors commented on the manuscript."

These errors have now been corrected in both the PDF and HTML versions of the article.

Published online: 30 May 2018

\footnotetext{
Correspondence: Kwanghee Lee (klee@gist.ac.kr) or M-H. Yoon (mhyoon@gist. ac.kr)

${ }^{1}$ School of Materials Science and Engineering, Gwangju Institute of Science and Technology, 123 Cheomdan-gwagiro, Buk-gu, Gwangju 61005, Republic of Korea

${ }^{2}$ Heeger Center for Advanced Materials and Research Institute for Solar and Sustainable Energies, Gwangju Institute of Science and Technology, 123 Cheomdan-gwagiro, Buk-gu, Gwangju 61005, Republic of Korea

${ }^{3}$ Department of Neurobiology, University of Chicago, Chicago, IL, USA

${ }^{4}$ Department of Robotics Engineering, Daegu Gyeongbuk Institute of Science and Technology, 333 Techno Jungang Daero, Hyeonpung-Myeon, Dalseonggun, Daegu 42988, Korea

${ }^{5}$ Present address: Laboratory of Organic Electronics, ITN, Linköping University, SE-601 74 Norrköping, Sweden
} changes were made. The images or other third party material in this article are included in the article's Creative Commons license, unless indicated otherwise in a credit line to the material. If material is not included in the article's Creative Commons license and your intended use is not permitted by statutory regulation or exceeds the permitted use, you will need to obtain permission directly from the copyright holder. To view a copy of this license, visit http://creativecommons.org/licenses/by/4.0/. 